IP Periodica Polytechnica Civil Engineering

\author{
62(2), pp. 398-403, 2018 \\ https://doi.org/10.3311/PPci.10917 \\ Creative Commons Attribution (1)
}

RESEARCH ARTICLE

\section{A Congestion Detection Based Traffic Control for Signalized Intersection}

\author{
István Varga $^{1 *}$
}

Received 21 April 2017; Accepted 26 October 2017

\begin{abstract}
The paper investigates a traffic-responsive control method applicable at isolated signalized intersections. The proposed strategy involves three basic parts: a traffic model, a reconfigurable regulator, and a congestion detection filter. Road traffic dynamics is modeled by the well-known store-and-forward approach. The controller is based on the efficient Linear Quadratic Regulator algorithm. The filter is designed by using the modified version (for discrete time case) of the Fundamental Problem of Residual Generation. The main achievement of the system is the ability to deal with a time-varying model parameter, namely the saturation flow rate of the road links. To this end, an error term is estimated continuously by appropriate fault detection algorithm. The predicted error term is further used by the reconfigurable controller which finally aims to mitigate the number of vehicles waiting at the stop line, i.e. the delay caused by the intersection. A simulation study is also carried out to demonstrate the effectiveness of the controller extended by congestion detection filter.
\end{abstract}

Keywords

road traffic control, congestion detection

\section{Introduction}

Road traffic congestion is a constant phenomenon everywhere in the world. Traffic networks of metropolitan areas frequently saturate during rush hours resulting in several direct and external impacts, e.g. time of delay, pollution, stress increase. The development of effective control strategies is therefore a real demand of our days.

The most effective control measure for road traffic is still performed via signal controllers by directly influencing the traffic dynamics. In the last decades, numerous solutions have been published in this field [10]. As a novel class among the investigated control solutions, one can highlight the achievements based on the modern control theory, e.g. [1, 5, 11-13]. These contributions intend to maximize the traffic capacity and mitigate vehicle queuing at the stop line, i.e. traffic-responsive controller logic is needed. Hence, the controller's capability for adaptivity is indispensable. This also means that the proper knowledge about the surrounding traffic states is critical. The better the quality of traffic detection is, the more effective the traffic control becomes $[2,16]$. However, the perfect measurement of all traffic variables is usually not possible or simply unrealistic due to financial reasons. Obviously, it is a trade-off problem.

Accordingly, a traffic signal strategy is introduced expanded by traffic state detection. An effective congestion detection filter is proposed for isolated intersection to enhance traffic control with a few number of detectors.

The proposed controller is able to modify the subsequent signal phase plan of the intersection in order to maximize the given cost function. The detected traffic state is the number of vehicles implying the cars unable to leave the link, i.e. link queue. This is a decision parameter for the intersection control which is a standard Linear Quadratic Regulator (LQR). The applied regulator is a reconfigurable controller capable to change its parameters depending on the current congestion information.

\section{Traffic modeling at intersection level}

The state space representation based urban traffic modeling (store-and-forward model) is widely used in literature, e.g. $[10,5,12]$. In this concept the state is defined as the number 
of vehicles and the control input as the green time of the given traffic direction. This approach, however, is limited concerning the saturation of the traffic network. The model is only valid for saturated traffic scenarios, i.e. when saturation flow rates are roughly constant. In case of undersaturated traffic, the positivity of state variable cannot be guaranteed in the model. On the other hand, oversaturated traffic may result in queue spill-back phenomenon which also corrupts the model. Accordingly, the main contribution to this model is an extension which is able to determine the number of vehicles capable to leave the link and the real number of cars which effectively left the stop line. This parameter will be further used within the controller scheme later.

\subsection{Store-and-forward traffic model}

The basic traffic model for road links applied for the control is represented by Fig. 1. This is based on the vehicle-conservation law, i.e. the number of vehicles in a given road link is always computable from the inflow and outflow traffic as long as they are known.

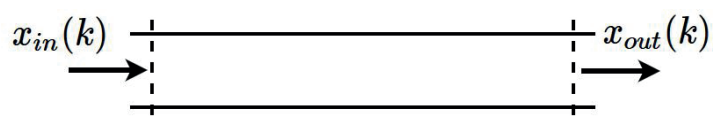

Fig. 1 Queue modeling on a signalized link

The vehicle-conservation equation can be simply defined in discrete time as follows:

$$
x(k+1)=x(k)+x_{\text {in }}(k)-x_{\text {out }}(k),
$$

where $x(k)$ denotes the number of vehicles within the link, $x_{i n}(k)$ the entering vehicles, and $x_{\text {out }}(k)$ the exiting vehicles, over the time period of $[k T,(k+1) T]$ with discrete time step index $k$ and sampling time $T$. If a traffic light is located at the downstream end of the link, this approach becomes the storeand-forward model [7] describing the traffic dynamics on signalized links. In this concept, exiting traffic $x_{\text {out }}(k)$ depends on the green time interval and also influenced by the saturation flow parameter $S$ which is defined as follows:

$$
S=\frac{n}{\sum t^{z}}
$$

where $\sum t^{z}$ denotes the sum of green times in one cycle $\left(T_{c}\right)$, and $n$ the number of vehicles leaving link $z$. In practice, $S$ is approximated by constant values according to Fig. 2 where $u$ denotes the effective green time interval which comprises the amber signal length beside the green signal. Typically, during the effective green time $S \approx 0.5$ (veh/s), otherwise zero. By using $S$ and $u$, Eq. (1) can be rewritten as:

$$
x(k+1)=x(k)+x_{i n}(k)-S u(k),
$$

which also means that

$$
x_{\text {out }}(k)=S u(k) .
$$

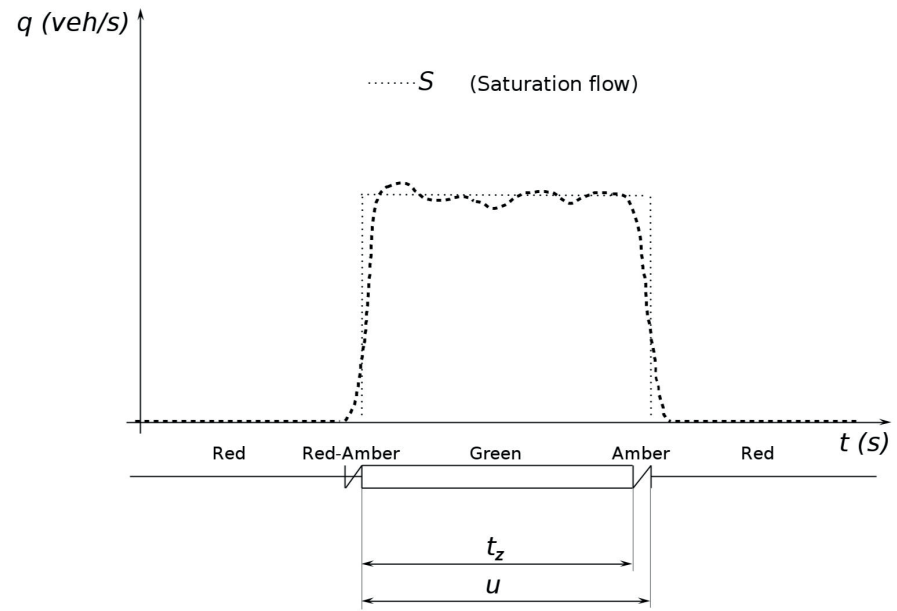

Fig. 2 Example for the saturation flow

\subsection{State space representation of the traffic dynamics}

In ideal case the number of vehicles on the link is given as follows:

$$
x_{\text {ideal }}(k)=S u(k) \text {. }
$$

Equation (5) is only valid in saturated traffic case, i.e. traffic is not over-saturated (congested) nor under-saturated (the assigned green time is longer than needed, only a part of the green time is exploited). Moreover, any slight disturbance might decrease the outflow. In fact, $x_{\text {ideal }}$ is equal to the number of effectively exited vehicles $\left(x_{\text {real }}\right)$ extended by a difference term $x_{f}$ :

$$
x_{\text {ideal }}(k)=x_{\text {real }}(k)+x_{f}(k) .
$$

In order to determine the value of $x_{f}$, a fault detection filter can be applied which is able to estimate the expected value of $x_{f}$ with relatively small error.

The fault detection filter design requires a state space representation form. The state variables of the system are defined as the number of waiting vehicles in each direction (link):

$$
x(k)=\left[x_{1}(k) x_{2}(k) x_{3}(k) x_{4}(k)\right]
$$

A discrete time state space representation is applied to model the traffic dynamics. Sample time $T$ is chosen to be equal to cycle time $T_{c}$ of the signal phase plan. The number of entering vehicles $x_{i n}(k)$ can be measured by standard vehicle sensor (e.g. loop or magnetic detector). However, sensors are not perfect. Hence, the measured input value $x_{i n}^{m}(k)$ is given as:

$$
x_{i n}^{m}(k)=x_{i n}(k)+v_{x}(k),
$$

where $v_{x}$ is a noise term.

According to Eq. (8), state equation (1) is formulated as:

$$
x(k+1)=x(k)+x_{\text {in }}^{m}(k)-x_{\text {out }}(k) .
$$

By substituting Eqs. (6) and (8) into Eq. (9), one arrives at the following difference equation:

$$
x(k+1)=x(k)+x_{i n}(k)+v_{x}(k)-S u(k)+x_{f}(k) .
$$


By using the standard LTI (linear time invariant) state space formula, state dynamics difference equation and measurement equation are given as:

$$
\begin{gathered}
x(k+1)=A x(k)+B u(k)+x_{i n}(k)+v_{x}(k)+x_{f}(k), \\
y(k)=C x(k)+v_{y}(k),
\end{gathered}
$$

where

$$
\begin{gathered}
A=C=I, \\
B=\left[\begin{array}{cccc}
-S_{1} & & & \\
& -S_{2} & & \\
& & \ddots & \\
& & & -S_{n}
\end{array}\right] .
\end{gathered}
$$

Matrix $C$ is identity matrix as it is assumed that the vehicle number is measurable directly on the links (e.g. by camera). $v_{y}(k)$ is considered as a Gaussian white noise relevant to the sensor.

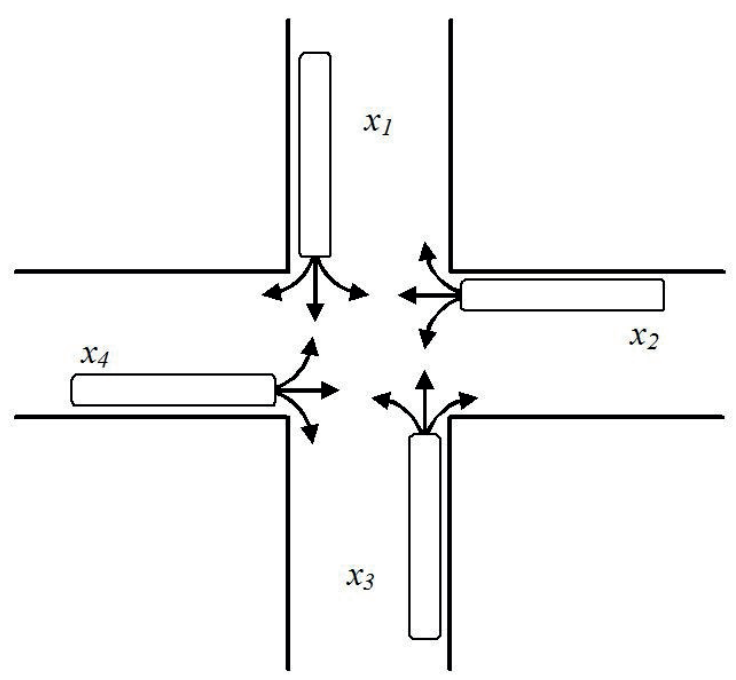

Fig. 3 Traffic model of a signalized intersection

In conclusion, the goal of the proposed controller is to minimize the number of vehicles waiting at the stop line, i.e. mitigate the delay time at the intersection (see Fig. 3). In accordance with Eq. (11) not all vehicles are capable to leave the junction in the given cycle time interval due to the following two reasons:

1. over-saturated traffic may result in queue spill-back phenomena which blocks exit flows;

2. the modeled saturation rate (Fig. 2) differs from that of the real dynamics, e.g. a bus or heavy goods vehicle starts with bigger time delay.

In case of the problem given in point 1) above, there exists a minimum number of vehicles capable to exit. This also indicates a minimum value for green time split:

$$
u_{i}(k+1)=\frac{B_{i, i}(k) u_{i}(k)-x_{f \mid i}(k)}{B_{i, i}(k)}, i=1,2, \ldots, n .
$$

This simply means that the given link must have a green time calculated by Eq. (15), i.e. a shortened time split is used by adapting to the blocked number of vehicles. This method is especially effective as the released time can be distributedto the other directions resulting in shortened queue lengths there.

To resolve the problem described by point 2), prolonged green time splits can be applied. This is obtained in two ways. On the one part, the feedback controller (explained in the sequel) per se minimizes the queue length by providing optimal green times. On the other part, $B$ (containing the saturation flow rates) can be considered as a time-varying matrix, calculated as follows:

$$
B_{i, i}(k+1)=\frac{B_{i, i}(k) u_{i}(k)-x_{f \mid i}(k)}{u_{i}(k)}, i=1,2, \ldots, n .
$$

The proposed controller is therefore a reconfigurable regulator, i.e. it is able to change their properties according to the actual congestion state. The controller structure is depicted by Fig. 4.

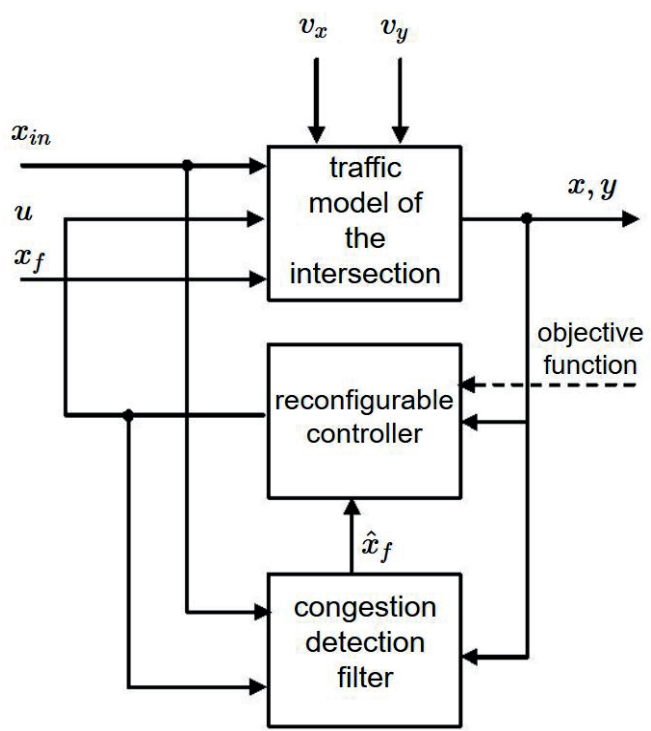

Fig. 4 Reconfigurable controller with congestion detection filter

\section{Congestion detection filter}

As described in the previous section a correction term $x_{f}$ is needed due to the characteristics of traffic dynamics at signalized intersection. Practically, $x_{f}$ represents an error term in the controller structure. Modern control theory applies fault detection filter technique to handle error terms. As $x_{f}$ refers to the evolution of congestion, the filter is named as congestion detection filter.

Fault detection filter is generally used in systems including safety critical functions. Therefore, these systems also contain fault diagnostic units. The detected errors within the controller imply effects which can be taken into consideration automatically in the closed-loop controlled system. Moreover, the error effect can be eliminated if the controller is reconfigured. This kind of modification means the reconfiguration of the whole system. Fault detection aims to recognize the errors by considering disturbance impacts as well $[3,4,6,14]$. 
The geometrical approaches to fault detection have been elaborated in the early $1970 \mathrm{~s}$. A significant geometrical approach of failure detection is called Fundamental Problem of Residual Generation (FPRG) [9]. FPRG also enables robust detection developed by [8]. FPRG filter method was chosen for the traffic control problem as well because it is able to detect failures separately in discrete time systems.

Recall Eq. (6). If residual $x_{f}$ is positive, then the link is congested. If $x_{f}$ is negative, more vehicles could exit than it was assumed previously by the model. Consider the following discrete time linear system with the error modeled as additive term:

$$
\begin{gathered}
x(k+1)=A x(k)+B u(k)+G v_{x}(k)+\sum_{i} L_{i} m_{i}(k) \\
y(k)=C x(k)+v_{y}(k),
\end{gathered}
$$

where

- $v_{x}(k)$ denotes the state and $v_{y}(k)$ the measurement bias assumed both to be Gaussian white noise with weighting matrix $G$ (they represent independent stochastic noises),

- $L_{i}$ is the direction of the additive error,

- $m_{i}(k)$ represents the input failure signal with $m_{i}(k) \in M$.

Congestion detection must be carried out for each queue length, i.e. for each direction of the intersection. Thus, the error directions relate to the given link queue. $L=\left[L_{1}, L_{2}, \ldots\right.$ , $L_{n}$ ] denotes the error directions with index $n$, the number of directions (also dimension of the system: $\operatorname{dim}(x)=n . L_{i}$ is unit vector, e.g. $L_{i}=[1,0, \ldots, 0]$.

It is noted that solution only exists in discrete time case if matrix $A$ is invertible and the system is observable [8]. If the previous conditions are fulfilled, the filter can be recast in a state observer form:

$$
\begin{gathered}
z(k+1)=F z(k)-E y(k)+D u(k) \\
r(k)=M z(k)-H y(k)
\end{gathered}
$$

where residual $r$ is the expected value of $, x_{f}, z$ is the state variable of the filter, and $F, E, D, M, H$ are parameter matrices for the FPRG filter design [15]. These matrices are calculated through a geometrical approach written in [8].

Based on Eq. (11), the intersection traffic model is reformulated:

$$
\begin{gathered}
x(k+1)=A x(k)+\underbrace{\left[\begin{array}{cc}
I & B
\end{array}\right]}_{\tilde{B}}\left[\begin{array}{c}
x_{i n}(k) \\
u(k)
\end{array}\right]+v_{x}(k)+x_{f}(k) \\
y(k)=C x_{k}+v_{y}(k)
\end{gathered}
$$

The FPRG filter also needs matrices $A, \tilde{B}, C$, and $L$ for fault detection [8].

\section{Traffic control method based on congestion detection filter}

Figure 5 depicts the controller's architecture. As observable the system consists of two regulator parts: a basic LQR and a supervisory algorithm. The supervisory controller is fed by the output of the fault detection filter (i.e. the values in vector $\left.\hat{x}_{f}(k)\right)$ and an objective function. Accordingly, Eqs. (15) or (16) are applied for the input signal calculation (optimal green time splits). If the objective function indicates the minimum length green time only, input signal is realized via Eq. (15) by disregarding the control input calculated with $\mathrm{LQR}$. If matrix $B$ must be modified, then Eq. (16) is used. In this case, optimal gain $K$ of LQR has to be also redefined by using the recalculated value of $B$.

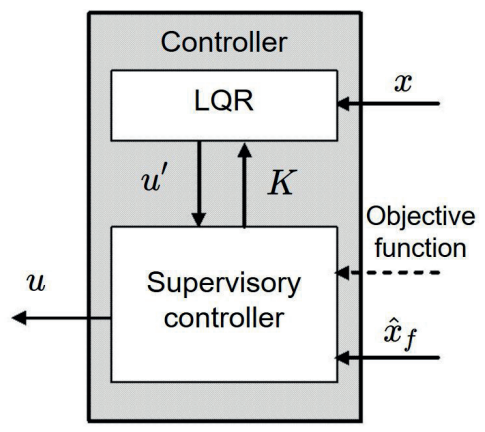

Fig. 5 The controller's architecture

The LQR control applied in this structure is formulated for the minimization of queue lengths in the network. By considering the measured vehicle numbers on the links, the regulator calculates optimal green time splits for each direction in the next discrete time step (practically the cycle time of the traffic controller: $T=T_{c}$ ). The quadratic functional of LQR is given in the regular form below.

$$
J=\frac{1}{2} \sum_{k=0}^{\infty}\left[x^{T}(k) Q x(k)+u^{T}(k) R u(k)\right],
$$

where $x$ is the state variable (vehicle numbers), $u$ is the control input (compasses green times for each direction), and $Q, R$ are appropriate diagonal weighting matrices. $u_{i}$ may represent the effective green time interval for link $i$, i.e. amber and green signals together. On the other hand, $u_{i}$ can be defined as a relative value expressing the difference compared to a nominal green time.

According to the theory of LQR, the controller minimizes the quadratic functional (22). The optimization is realized through state feedback control based on the calculated LQ gain $K$. The green time splits are given therefore as follows:

$$
u(k)=-K(k) x(k)+u_{\text {nom }}(k),
$$

where $u_{\text {nom }}$ denotes the nominal green time as a reference signal if applicable.

Tuning matrices $Q$ and $R$ strongly influence the quality of the control. A reasonable approach is to set $Q=\operatorname{diag}\left(\frac{1}{\left(x_{i}^{\max }\right)^{2}}\right)$ [10]. $R$ might be valued as the variance of the measured output $y$.

The realization of the LQR algorithm together with the fault detection filter is straightforward based on the literature. At the same time, in case of real-world application basic rules of traffic engineering must be considered as well, i.e. the green time splits are restricted to be within an interval: 


$$
\begin{gathered}
u_{i}^{\min } \leq u_{i}(k) \leq u_{i}^{\max } \quad i=1,2, \ldots, n, \\
\sum_{i=1}^{N} u_{i}(k)+T_{\text {lost }}=T_{c},
\end{gathered}
$$

where $T_{\text {lost }}$ is the lost time of the signalization (when no vehicle movement is allowed in the intersection). Moreover, $u_{i}$ must be integer as traffic light visualize signals in time unit of seconds. $T_{c}$ and $T_{\text {lost }}$ are usually fixed values. Constraints given by (24) can be carried into effect via appropriate linear programming running after the controller's calculation and before the real action of signaling in the next cycle.

\section{Simulation based evaluation}

A simulation based example is provided to test the intersection control with congestion detection filter. The simulation was realized in MATLAB Simulink environment. The goals of simulation are twofold:

1. show the filter's ability to detect congestion;

2. justify the efficiency of the reconfigurable controller by using the congestion information.

Practically, the traffic dynamics depicted by Fig. 3 was simulated with the following parameters:

- 4 directions,

- cycle time of the signal plan is chosen as $T_{C}=60 \mathrm{sec}$,

- control (sample) time is $T=T_{C}$,

- the vehicle input to the system $\left(x_{i n}\right)$ was modeled as normally distributed random variable,

- simulation time period was $3600 \mathrm{sec}$.

First the results of the congestion detection filter are presented. In order to evaluate the filter, congestion was simulated as follows:

- at each cycle one vehicle gets stuck in direction 1 ,

- at each cycle two vehicles get stuck in direction 2 .

This means that the fault term was set to $x_{f}=[1,2,0,0]^{T}$. The filter results concerning each direction are plotted in Figs. 6-7.

It can be seen that the filter efficiently detected the number of vehicles $\left(x_{f}(k)\right)$ which were not able to exit under the given green time splits. The performance of the filter strongly depends on the measurement noise $\left(v_{y}\right)$, the variance of $x_{i n}$, as well as the tuning of the detection filter.

Beside the filter performance also the reconfigurable controller was tested in the simulation environment. In this case, the simulation period was set to 30 minutes. The proposed traffic-responsive controller was therefore compared to a regulator based on fixed-time signal plan. In the simulation two vehicles get stuck in direction 2 at each cycle, i.e. the fault term was set to $x_{f}=[1,2,0,0]^{T}$. This resulted in a higher queue length of that link accordingly. The queue lengths of the intersection in case of fixed-time signal control are depicted in Figs. 8-9.

At the same time, the reconfigurable controller extended by congestion detection filter was able to efficiently attenuate the jam evolution in direction 2. The queue lengths of the intersection in case of reconfigurable control are depicted in Figs. 10-11.
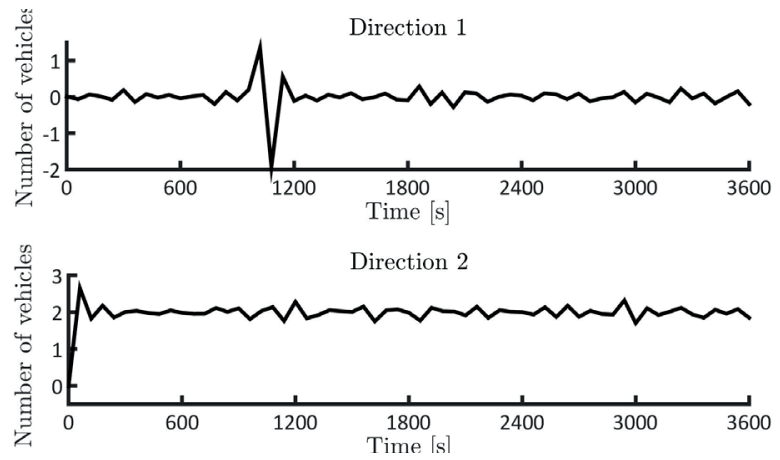

Fig. 6 The results of the congestion detection filter in direction 1 and 2
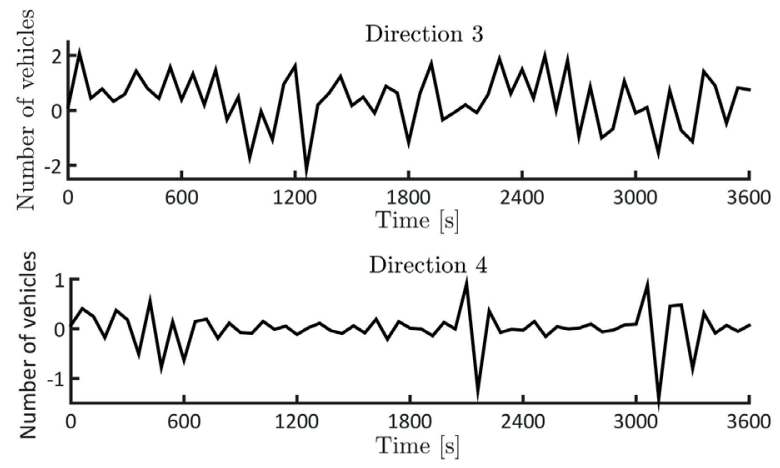

Fig. 7 The results of the congestion detection filter in direction 3 and 4
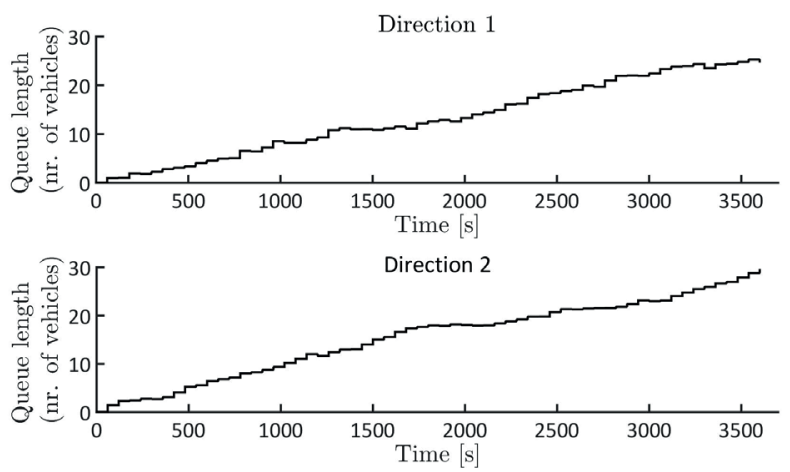

Fig. 8 Vehicle number in direction 1 and 2 in case of fixed-time control

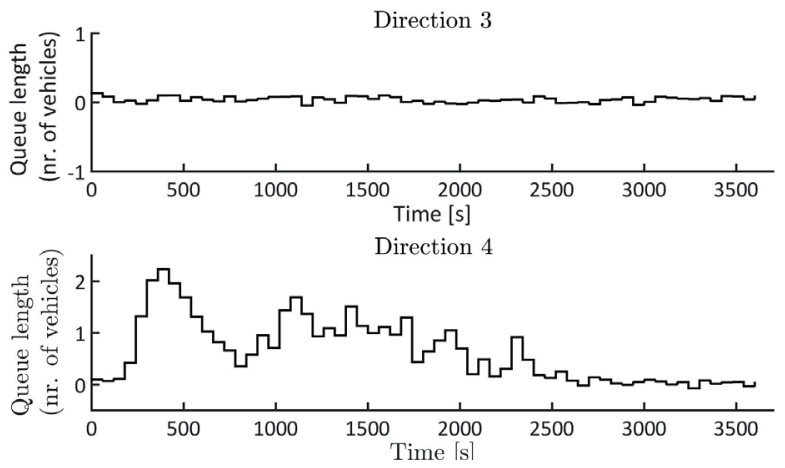

Fig. 9 Vehicle number in direction 3 and 4 in case of fixed-time control

Although the longer green times for direction 2 regulated by the reconfigurable controller were calculated at the other directions' expense, the sum of all vehicles waiting at the stop lines of the junction was higher by $23 \%$ finally. Furthermore, the distribution of these queues among the directions was much smoother compared to the fixed-time case. 

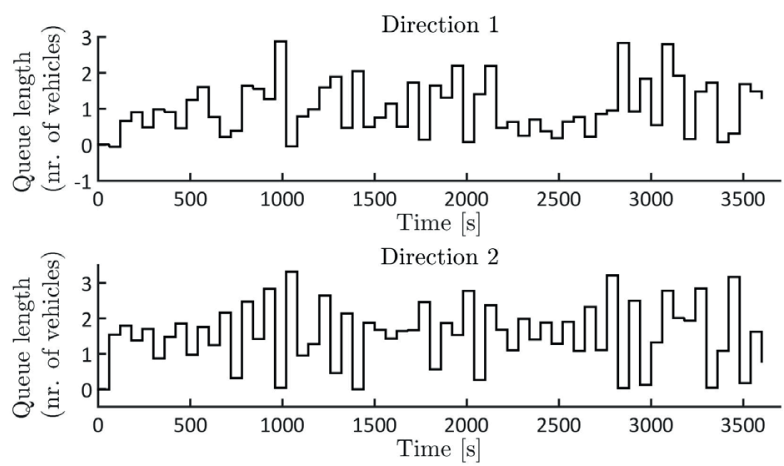

Fig. 10 Vehicle number in direction 1 and 2 in case of reconfigurable control
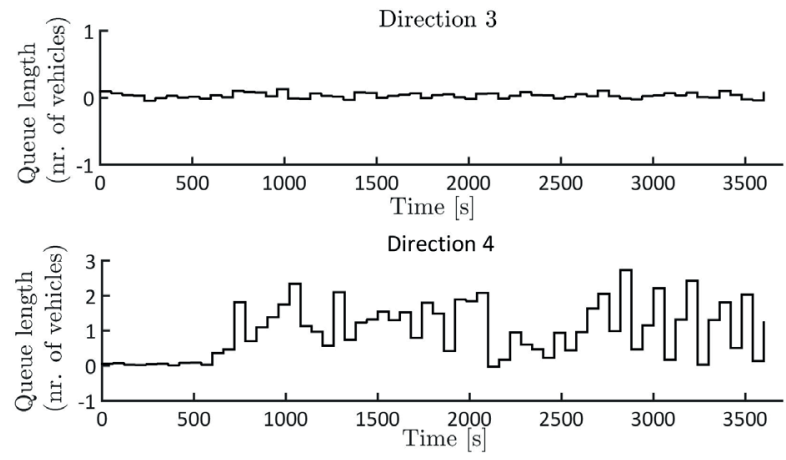

Fig. 11 Vehicle number in direction 3 and 4 in case of reconfigurable control

The result therefore demonstrates that the proposed method can be efficiently applied as a real-time traffic control candidate for signalized intersection.

\section{Conclusions}

Based on the theory of FPRG fault detection filter an algorithm for congestion detection has been elaborated for signalized intersection. The congestion detection filter is able to recognize the deviation from the nominal traffic model. By using this information, the traffic light controller can change a critical model parameter (saturation flow rate) and recalculate the signal phase plan at each cycle.

A simulation example has also been introduced to demonstrate the proposed control concept. Simulation results proved that the congestion detection filter can efficiently help the reconfigurable controller performing more reliable traffic signal control. Practically, an adaptive control can be realized intending to mitigate the queue lengths of the junction.

In conclusion, it has been shown that the congestion detection filter based strategy is an appropriate choice for traffic control in isolated signalized intersection. Future research work consists of extending the method for multiple junctions, i.e. for urban road traffic network.

\section{Acknowledgement}

The author would like to thank Tamás Tettamanti and Balázs Kulcsár for their valuable help.

\section{References}

[1] Aboudolas, K., Papageorgiou, M., Kosmatopoulos, E. "Store-and-forward based methods for the signal control problem in large-scale congested urban road networks". Transportation Research Part C: Emerging Technologies, 17(2), pp. 163-174. 2009. https://doi.org/10.1016/j. trc.2008.10.002

[2] Asaithambi, G., Sekar Mourie, H, Sivanandan, R. "Passenger car unit estimation at signalized intersection for non-lane based mixed traffic using microscopic simulation model". Periodica Polytechnica Transportation Engineering, 45(1), pp. 12-20, 2017. https://doi.org/10.3311/ PPtr.8986

[3] Bokor, J., Balas, G. J. "Detection filter design for LPV systems - a geometric approach". Automatica, 40(3), pp. 511-518. 2004. https://doi. org/10.1016/j.automatica.2003.11.003

[4] Bokor, J., Shinar, J., Kulcsár, B. "On the detection of unknown input in positional control problems with noisy measurements". In: Proceedings of 16th IFAC World Congress, Vol. 38, pp. 802-807, 2005.

[5] Diakaki, C., Papageorgiou, M., Aboudolas, K. "Traffic-responsive urban network control using multivariable regulators". In: International Conference on Modeling and Management in Transportation, Vol. 2, pp. 11-16, Poznan/Cracow, 1999.

[6] Edelmayer, A., Bokor, J., Keviczky, L. "An Hळ filtering approach to robust detection of failures in dynamical systems". In: Proceedings of 33rd IEEE Conference on Decision and Control, pp. 3037-3039, 1994.

[7] Gazis, D. C., Potts, R. B. "The oversaturated intersection". In: Proceedings of the Second International Symposium on Traffic Theory, London, UK, pp. 221-237, 1963.

[8] Kulcsár, B. "Design of robust detection filter and fault correction controller". PhD thesis, Budapest University of Technology and Economics, 2005.

[9] Massoumnia, M. A. "A geometric approach to the synthesis of failure detection filters". IEEE Transactions on Automatic Control, 31(9), pp. 839-846. 1986. https://doi.org/10.1109/TAC.1986.1104419

[10] Papageorgiou, M., Diakaki, C., Dinopoulou, V., Kotsialos, A., Wang, Y. "Review of road traffic control strategies". Proceedings of the IEEE, 91(12), pp. 2043-2067. 2003. https://doi.org/10.1109/ JPROC.2003.819610

[11] Tettamanti, T., Varga, I. "Traffic control designing using model predictive control in a high congestion traffic area". Periodica Polytechnica Transportation Engineering, 37(1-2), pp. 3-8. 2009. https://doi. org/10.3311/pp.tr.2009-1-2.01

[12] Tettamanti, T., Varga, I., Kulcsár, B., Bokor, J. "Model predictive control in urban traffic network management". In: 16th Mediterranean Conference on Control and Automation, Ajaccio, Corsica, France, 2008, pp. 1538-1543, 2008. https://doi.org/10.1109/MED.2008.4602084

[13] Tettamanti, T., Varga, I., Péni, T. "MPC in urban traffic management". In: Zheng, T. (ed.) Model Predictive Control, (pp. 251-268.) InTech, 2010. https://doi.org/10.5772/9922

[14] Tyler, M., Asano, K., Morari, M. "Application of moving horizon estimation based fault detection to cold tandem steel mill". Technical report, ETH, 1996.

[15] Varga, I. "Közúti folyamatok paramétereinek modell alapú becslése és forgalomfüggő irányítása". PhD thesis, Budapesti Műszaki és Gazdaságtudományi Egyetem, 2006. (in Hungarian)

[16] Zefreh, M. M., Török, Á., Mészáros, F. "Average vehicles length in two-lane urban roads: A case study in Budapest". Periodica Polytechnica Transportation Engineering, 45(4), pp. 218-222, 2017. https://doi. org/10.3311/PPtr.10744 